\title{
Collaborative Sketching with Distributed Displays and Multimodal Interfaces
}

\author{
Florian Geyer, Hans-Christian Jetter, Ulrike Pfeil, and Harald Reiterer \\ University of Konstanz \\ Human-Computer Interaction Group \\ Box D73, 78457 Konstanz, Germany \\ \{firstname.lastname\}@uni-konstanz.de
}

\begin{abstract}
In this paper we describe a system design for supporting creative group activities using distributed displays and multimodal interaction. We describe the rationale behind our approach and proposed interaction techniques for supporting collaborative sketching. Our goal is to understand how the interface metaphor, display space and interaction modalities may influence exploration and communication in collaborative settings.
\end{abstract}

ACM Classification: H5.2 [Information interfaces and presentation]: User Interfaces. - Graphical user interfaces.

General terms: Design, Experimentation

Keywords: Creativity support, Design tools, Collaboration, Multi-display environments, Multimodal interaction

\section{INTRODUCTION}

When designers are creating and revising ideas during early design stages, they often harness the spatial properties of the environment [1]. Design sketches are shared on large whiteboards or spread out on tables and walls for comparison and discussion with other designers. In design studio environments, the room itself is an important tool that allows organizing information in an informal and spatial way. Especially during group activities, accessibility and visibility of artifacts is crucial for efficient communication. This is one of the reasons why designers often prefer to work with pen and paper during creative group activities [2]. Paper provides a private workspace while sharing is as simple as pinning the paper on the wall or spreading it out on the table. However, when using desktop-based electronic tools, the artifacts that are created are often hidden in file systems. At the same time, multiple devices are required to work simultaneously in group settings. Even when using electronic whiteboard systems, exchanging sketches to other displays is awkward and can lead to breakdowns of the crea- tive flow. In the following, we examine existing approaches to these issues before describing the rationale and proposed interaction techniques of our alternative approach.

\section{EXISTING APPROACHES}

i-LAND [3] is a smart environment supporting creative problem solving activities within groups. It allows accessing and transferring digital artifacts between different devices, like whiteboards, tables and mobile displays. Transferring objects is realized by drag \& drop gestures across devices or by saving digital artifacts onto a physical token that can be accessed from retrieval stations. In Team Storm [4], designers can cooperatively sketch ideas with a system that combines multiple tablet devices (focus displays) with an electronic whiteboard (context display). Individual sketches can be transferred from the tablets to the whiteboard for discussion via a shared workspace window. Sketches can also be transferred back to the tablets for annotation by individuals. Both approaches support efficient collaboration for sketching in early design phases. Nevertheless, we aim to extend these approaches by investigating the effects of the overall interface metaphor, display space as well as interaction modalities on exploration and communication during different group activities.

\section{RESEARCH GOALS}

We think that the spatial and physical properties of the workspace are crucial for effective collaboration in creative group work. We therefore emphasize on supporting (a) informal spatial information management by providing flexible display space for different situations and activities. This includes both the (b) design of the overall interface metaphor as well as the (c) design of the physical work environment. As we think that the properties of physical artifacts contribute to creative thinking, we also seek to (d) improve ad-hoc accessibility of the shared information by making the digital representations more tangible. Nevertheless, instead of the need to transfer content between devices and displays, we think that (e) one common workspace across multiple distributed displays may provide benefits for improving group awareness. Finally, we aim on (f) supporting natural interaction modalities, like touch and pen on different display sizes and display types in order to support a variety of creative work styles suitable for flexible group compositions and alternating activities like colla- 

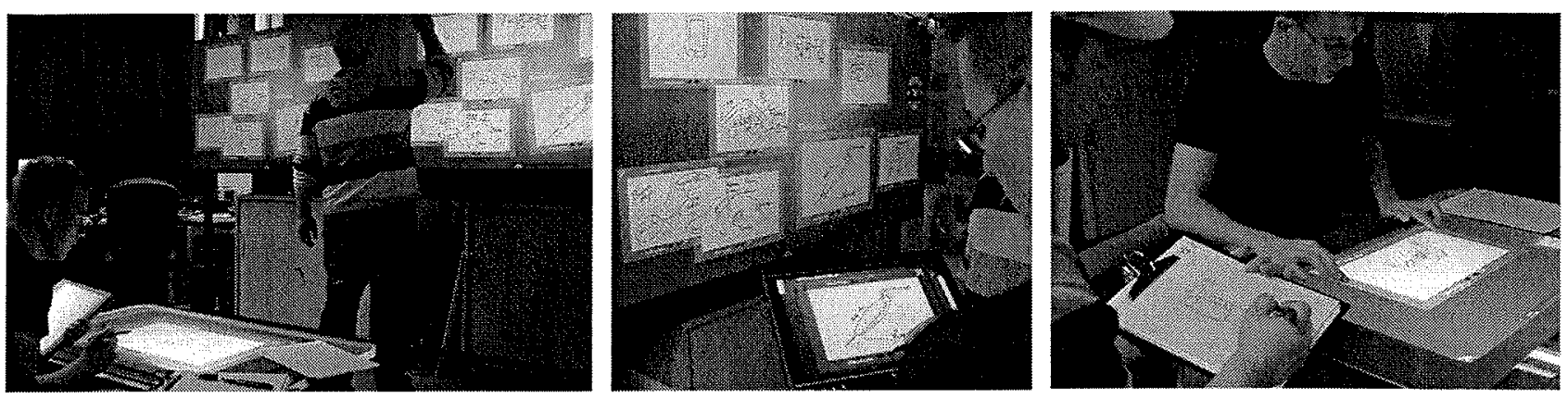

Figure 1: Design studio setting (a), focus and context navigation by remote zooming actions (b), digital pen and paper (c)

borative sketching, discussions and presentations, private reflective work or distributed feedback sessions.

\section{TOOL DESIGN}

Since our approach emphasizes on supporting the spatial properties of creative design practice, we decided to employ a zoomable pin board as the overall interface metaphor [5]. Sketches on the pin board can be scaled, rotated and moved. This allows designers to arrange the digital representations in a similar way as on physical display walls. The pin board can also be navigated by zooming manipulations. Thereby, the view onto the contents of the whiteboard can be changed according to the current point of interest. Zooming actions can also be triggered remotely across different devices. Manipulations and annotations however are synchronized over a central database repository to keep all contents consistent. Additionally, we design our tool around a physical environment that resembles a digital design studio by providing extensive display space on one large interactive wall with pen interaction, two multi-touch tabletops and multiple tablet PCs (see Figure 1, a). The pin board interface spreads across all these displays providing independent views on the same content. These views can also be used as focus and context displays (see Figure 1, b)

We think that different activities should be supported by suitable modalities. Collaborative tasks like sorting existing sketches and focusing on sketches during discussions work well on tabletops because multi-touch interaction provides an efficient modality for this task. However, cooperatively creating content is better done at the interactive whiteboard as it offers more display space and more precise pen input. However, for individual reasoning, private displays are preferred. This is also the reason why we decided to integrate mobile tablets and digital pen and paper into our system (see Figure 1, b,c). Tablets can be used for individual sketching, browsing and annotation. Digital pen \& paper adds the natural modality of physical paper to our workspace. New sketches can be created by simply grabbing a blank sheet of paper while existing digital sketches can be printed out for individual activities like reflection, distributed reviews or annotation. Sketches made on paper are instantly transferred via Bluetooth connections to the digital representations on the pin board interface. Synchronization then updates all distributed displays. By supporting multiple digital pens at the same time, we can extend the capacity for parallel input. This allows for real-time collaboration awareness, even when designers work on regular paper. Thus, our system enables parallel work and supports different modalities, by still providing the same basic interface metaphor. This allows users to flexibly choose an adequate modality for the current activity without the need to transfer content from one screen to another.

\section{FUTURE WORK}

We are currently extending the system with groupware features. This includes an automatic locking mechanism of objects that are currently manipulated to cope with concurrency problems. In addition, we are also adding a location tracking system for identification of users and their display association. In future work, we want to integrate this metadata for analytical visualizations of design sessions and for presenting passive information on displays that are not actively used by the group.

\section{REFERENCES}

1. Vyas D., Veer G.V., Heylen D., Nijholt A. Space as a Resource in Creative Design Practices. In Proceedings of INTERACT'09, LNCS, Springer, 2009, pp. 169-172.

2. Cook D., Bailey B. Designers' Use of Paper and the Implications for Informal Tools. In Proceedings of OZCHI'05, CHISIG, AU, 2005. pp. 1-10.

3. Streitz N. A., Geißler J., Holmer T, et al, i-LAND: An Interactive Landscape for Creativity and Innovation. In Proceedings of CHI'99, SIGCHI, ACM Press, NY, US. 1999. pp. 120-127.

4. Hailpern J., Hinterbichler E., Leppert C., et al. TEAM STORM: Demonstrating an Interaction Model for Working with Multiple Ideas During Creative Group Work. In Proceedings of $\mathrm{C}^{2} \mathrm{C}^{\prime} 07, \mathrm{ACM}$ Press, NY, US. 2007. pp. 193-202.

5. Jetter, H-C., Gerken, J., Zöllner, M., Reiterer, H. Model-based Design and Prototyping of Interactive Spaces for Information Interaction. To appear in Proceedings of $H C S E^{\prime} 10$, Springer. 2010. 